\title{
Multiple myeloma cell-derived microvesicles are enriched in CD147 expression and enhance tumor cell proliferation
}

\author{
Bonnie K. Arendt ${ }^{1}$, Denise K. Walters ${ }^{1}$, Xiaosheng Wu ${ }^{1}$, Renee C. Tschumper ${ }^{1}$ and \\ Diane F. Jelinek ${ }^{1,2}$ \\ ${ }^{1}$ Department of Immunology, Mayo Clinic, College of Medicine, Rochester, MN \\ 2 Division of Hematology, Department of Medicine, Mayo Clinic, College of Medicine, Rochester, MN \\ Correspondence to: Diane F. Jelinek, email: jelinek.diane@mayo.edu \\ Keywords: MPTOG030, PKC $\delta$, E-cadherin, HDAC, differentiation \\ Received: June 20, $2014 \quad$ Accepted: July 2, $2014 \quad$ Published: July 3, 2014
}

This is an open-access article distributed under the terms of the Creative Commons Attribution License, which permits unrestricted use, distribution, and reproduction in any medium, provided the original author and source are credited.

\section{ABSTRACT}

Multiple myeloma (MM) is characterized by the clonal expansion of malignant plasma cells within the bone marrow. There is a growing literature that tumor cells release biologically active microvesicles (MVs) that modify both local and distant microenvironments. In this study, our goals were to determine if MM cells release MVs, and if so, begin to characterize their biologic activity. Herein we present clear evidence that not only do both patient MM cells and human MM cell lines (HMCLs) release MVs, but that these MVs stimulate MM cell growth. Of interest, MM-derived MVs were enriched with the biologically active form of CD147, a transmembrane molecule previously shown by us to be crucial for MM cell proliferation. Using MVs isolated from HMCLs stably transfected with a CD147-GFP fusion construct (CD147 ${ }^{\text {GFP }}$ ), we observed binding and internalization of MV-derived CD147 with HMCLs. Cells with greater CD147GFP internalization proliferated at a higher rate than did cells with less CD147 ${ }^{\text {GFP }}$ association. Lastly, MVs obtained from CD147 downregulated HMCLs were attenuated in their ability to stimulate HMCL proliferation. In summary, this study demonstrates the significance of MV shedding and MV-mediated intercellular communication on malignant plasma cell proliferation, and identifies the role of MVenriched CD147 in this process.

\section{INTRODUCTION}

Multiple myeloma (MM) remains a largely incurable neoplasm defined by the presence of a clonal expansion of malignant plasma cells (PC) in the bone marrow (BM), $\geq 3 \mathrm{~g} / \mathrm{dL}$ of monoclonal immunoglobulin (Ig; M-protein), and the presence of end organ damage which may include lytic bone lesions [1]. Monoclonal gammopathy of undetermined significance (MGUS) and smoldering (SMM) are the asymptomatic stages which precede MM, with MGUS being considered the premalignant stage [2]. Although the clonal PC populations in both asymptomatic conditions may remain remarkably stable for years, MGUS and SMM patients have a significant, life-long increased risk of progressing to overt $\mathrm{MM}$ at a rate of $1 \%$ and $10 \%$ per year, respectively $[2,3]$. While there have been considerable advances in treatment of this disease, the median survival of MM patients still remains only 4-7 years [4]

Over the last decade, significant investigation in the MM field has demonstrated that the interaction of malignant PCs with BM stromal cells within the microenvironment is essential for survival of the $\mathrm{PC}$ clone $[5,6]$. The observation of this intercellular communication and that MM cells modify the microenvironment is highly suggestive that one of the first biologically relevant changes necessary for progression of MGUS or SMM to $\mathrm{MM}$ is for a member(s) of the clone to evolve and acquire the molecular changes needed to reprogram its immediate microenvironment into a supportive, growth-promoting microenvironment. Microvesicles (MVs) are increasingly recognized as mediators of intercellular communication due to their capacity to merge with and transfer a collection of bioactive molecules to recipient cells [7]. MVs are cellderived particles that are spontaneously shed by a variety of cell types under normal physiological conditions.[7] 
Notably, tumor cells have been shown to shed MVs in an increased, dysregulated $[8,9]$ fashion, resulting in the presence of circulating MVs in patients with various types of cancers $[10,11]$. MVs range in size from $0.05-1.0 \mu \mathrm{m}$ and formation occurs through the outward budding and fission of the plasma membrane which can be induced by multiple events including the activation of PKC and/or increased intracellular calcium $\left(\mathrm{Ca}^{2+}\right)$ concentrations [12, 13]. Studies have shown that the molecular and genetic contents of MVs are very specific rather than a random sampling of all molecules expressed in the originating cell [14] and MV release is thought to be dynamic and dependent on both the cell type and activation status of the cell from which they are released [15]. Compelling evidence suggests a role for tumor-derived MV-mediated modulation of cells within the tumor microenvironment of various malignancies, including lung, breast, melanoma, and CLL [16-19]. Moreover, MV-modulation activity has been shown to correlate with tumor cell invasiveness resulting in angiogenesis and metastasis.

Despite the significant number of studies showing a role for TDMVs in various solid tumors, to date there has been very little study of TDMVs in MM. To our knowledge, there have been only three published studies of TDMVs and MM, and the scope in each case was extremely limited. One group studied the effect of serum deprivation on the size of MVs released by two HMCLs [20] and in a separate paper, examined the ability of MVs from one HMCL to stimulate endothelial cell proliferation [21]. The second group performed proteomic analysis of MVs released by two HMCLs and compared the profiles with intact cells [22]. Although these papers demonstrate MM cell release of MVs, current knowledge of the biological activity of MM cell derived MVs remains very limited.

Regarding a potential biological role for MM cell derived MVs, the CD147 molecule has been shown to be present on MVs isolated from lung and ovarian cancer cell lines $[23,24]$. We were the first to show that CD147 is upregulated in MM cells and appears necessary for tumor cell growth. In addition, expression levels of CD147 increase during MM progression [25]. CD147, also known as extracellular matrix metalloproteinase (MMP) inducer (EMMPRIN), is a transmembrane glycoprotein ubiquitously expressed at a low level on the surface of many different cell types [26]. Increased CD147 expression is biologically relevant because of its ability to increase angiogenesis [27], tumor growth [28], and MMP production leading to extracellular matrix degradation [28, 29]. Based on these findings as well as the growing body of literature regarding the role of MVs in intercellular communication, the aim of the present study was to continue our research of CD147 by investigating its potential involvement in the disease as a constituent of MM cell released MVs. Our data clearly demonstrate that malignant PCs shed bioactive MVs, and that this bioactivity can partially be attributed to the presence of CD147 within these MVs.

\section{RESULTS}

\section{Analysis of MM cell-derived MVs}

Although cell-to-cell contact and subsequent secretion of soluble factors has long been known to facilitate communication within the tumor microenvironment, recent evidence suggests that these factors can also be released via membrane-bound specialized vesicles which contain cellular contents that aid intercellular communication. Although there is emerging evidence that HMCLs release MVs, it remains unknown if primary patient MM cells release MVs, and if so, does this differ from normal BM PCs. To address these questions, we first used SEM to analyze HMCLs, primary patient MM cells, and normal BM PCs for evidence of cell surface associated MVs. Figure 1A demonstrates that the surface of representative HMCLs was rich with MVs both spherical and villi-like in appearance and that primary patient MM PCs showed similar structures, effectively demonstrating MV projections around the cell membrane

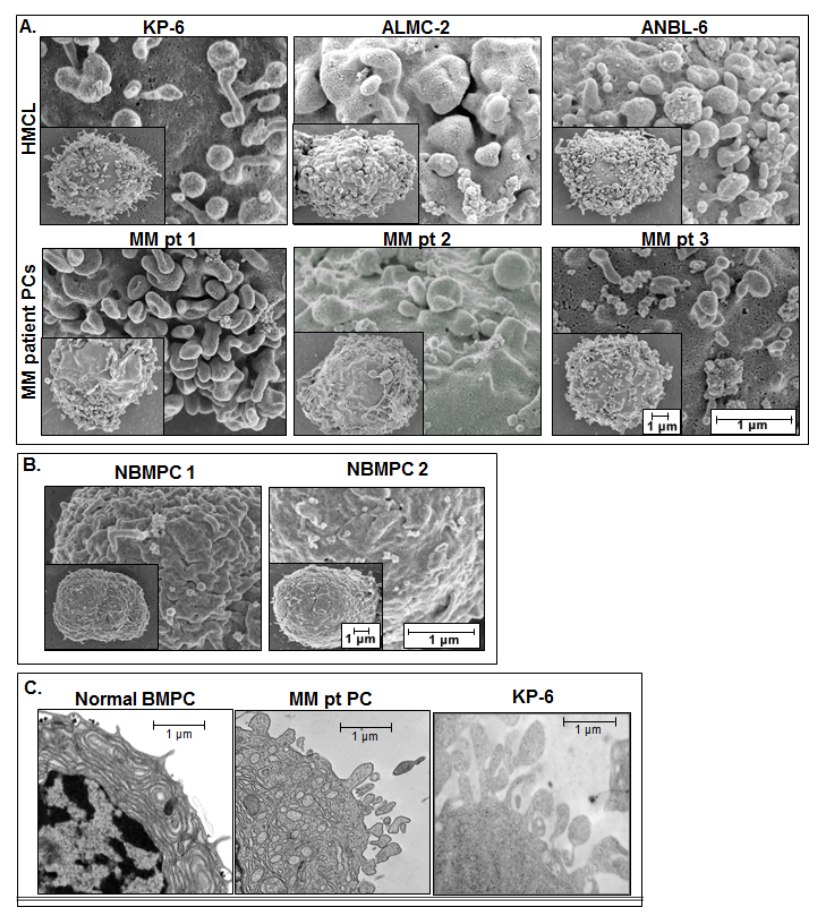

Figure 1: EM analysis of surface associated MVs. A) MV production as revealed by SEM on 3 HMCLs and 3 primary patient $\mathrm{CD} 138^{+} \mathrm{MM}$ cell samples. Whole cell images are shown in the lower left of each panel and the scales for each image are shown in the bottom right panel (MM pt 3). B) SEM analysis of 2 normal bone marrow PC samples. C) Surface MV comparison by TEM of normal bone marrow PC (left), MM patient PC (middle), and the KP-6 HMCL (right). 
periphery. Moreover, the size of these surface structures ranged from $\sim 0.05-1.0 \mu \mathrm{m}$ in diameter in both HMCLs and patient MM cells and is therefore consistent with the published range of MVs [30-32]. In striking contrast, SEM analysis of normal BM PCs revealed a remarkably smooth cell surface (Figure 1B). We also used TEM and the results shown in Figure 1C support the SEM data, with normal BM PC cell surfaces exhibiting fewer MVs than MM PCs and the KP-6 HMCL.

To verify that the apparent surface associated MVs were indeed being released we collected conditioned media from several HMCLs and processed it as described in the Materials and Methods. The size of the MVs was analyzed using several complementary methods. We first used flow cytometry and submicron sizing beads and the size of HMCL derived MVs was consistent with the reported size range of MVs (Figure 2A). Because it is known that some MVs are too small to be detected by flow cytometry [33], we also performed Nanoparticle Tracking Analysis to independently characterize the size of MMderived MVs. Figure 2B shows a representative analysis of MV size using this technology and, as expected, this method permitted demonstration of a significant number of MVs too small to be detected by flow cytometry. Third, we also used TEM to assess size. Figures $2 \mathrm{C}$ and 2D demonstrate the spherical structure of MVs isolated from the HMCLs RM43 and ALMC-2, respectively. This analysis also demonstrates the range in size of MM cell derived MVs.

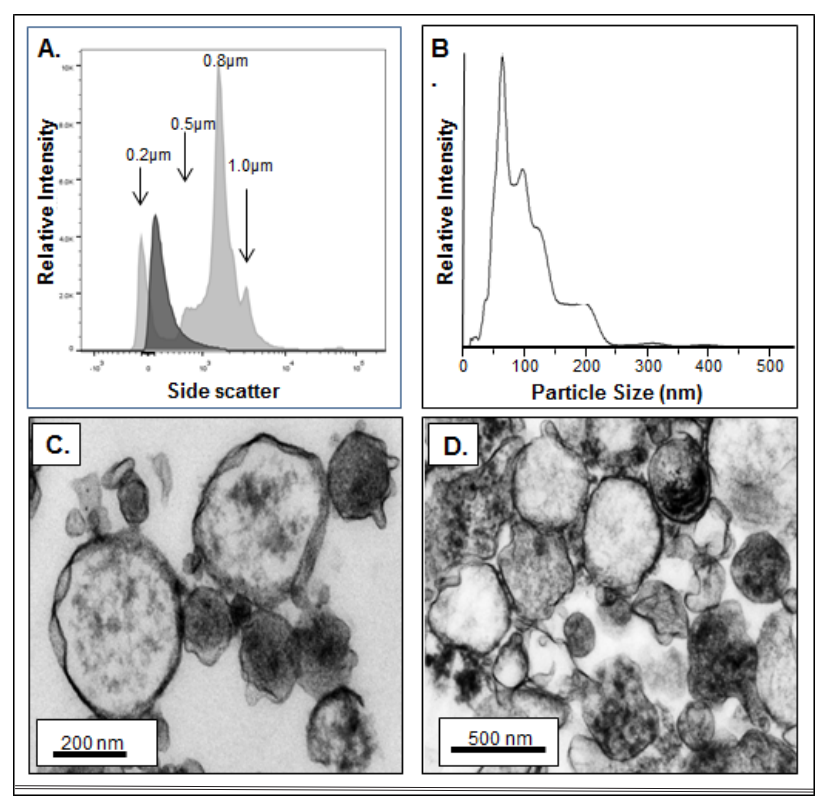

Figure 2: MM-derived MV dimensions. A) Histogram showing by flow cytometry the size of ALMC-2 derived annexin-V+ MVs (dark gray histogram) in comparison with 0.2 , $0.5,0.8$ and $1.0 \mu \mathrm{m}$ calibration beads (light gray histograms). B) Nanoparticle tracking analysis of MVs isolated from purified MM patient plasma cells. C, D) TEM of RM43 and ALMC-2 HMCL MVs, respectively.

\section{MM-derived MVs are annexin- $\mathrm{V}+$ and express full-length CD147}

The images shown in Figure 2 support our conclusion that MM cells release MVs. However, MVs are also defined as being annexin- $\mathrm{V}+$. Therefore, we next assessed annexin- $\mathrm{V}$ expression on HMCL-derived MVs by flow cytometry, and given our prior work on CD147 in MM [25] and reports in the literature that CD147 is present on MVs isolated from lung and ovarian cancer cell lines [23, 24], we also assessed MM-derived MV CD147 expression. As shown in Figure 3A, MVs isolated from 3 representative HMCLs were indeed all annexin- $\mathrm{V}+$; the MVs were also clearly CD147 positive (Fig. 3A, right histograms). To further investigate MV CD147 expression, we used IEM. Figure 3B shows CD147 expression on released and cell surface associated MVs on the ALMC-2
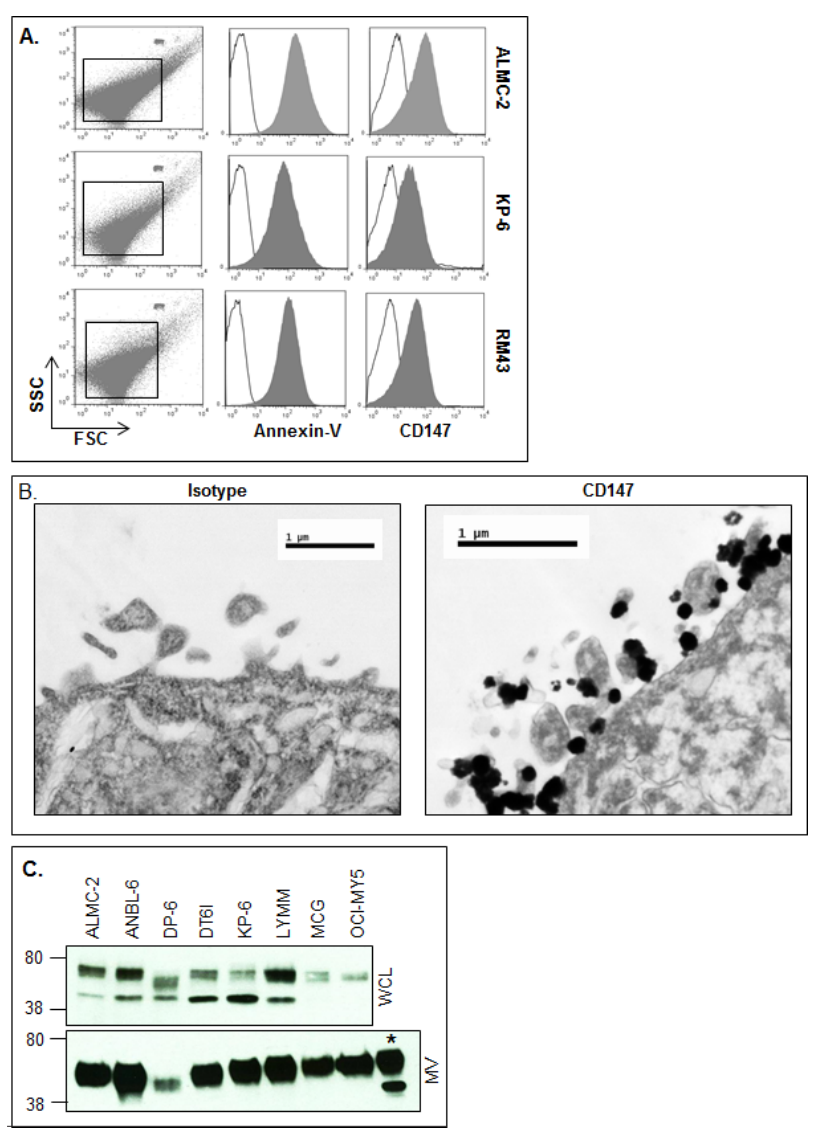

Figure 3: HMCLs shed MVs are enriched for CD147. A) Flow cytometric analysis of HMCL-derived MVs stained with MV marker annexin-V. CD147 staining identifies the molecule as a component within HMCL MVs (shaded histogram) as compared to the isotype control (open histogram). $1 \mu \mathrm{M}$ beads used for size verification. B) IEM employing immunegold particles to view CD147 expressing ALMC-2 MVs (right panel) as compared to the isotype-matched control (left panel). C) Western blotting of HMCL panel for CD147 in $5 \mu \mathrm{g}$ MV lysates (lower panel) and whole cell lysates (WCL; upper panel). Asterisk (*) denotes ALMC-2 WCLs used as a positive control. 
cell line.

Because the CD147 molecule can be cleaved from cell surfaces [34], it was next necessary to characterize the molecular size of MV-associated CD147. To accomplish this, we used western blot analysis to probe the size of MV-associated CD147 vs. intact cell membrane CD147. We also used a CD147 antibody that recognizes the intracellular domain (C-terminus) thereby only permitting detection of transmembrane CD147. As we have previously shown [25], when whole cell lysates are probed for CD147, there are two dominant species observed corresponding to low and high glycosylated forms of CD147 (Fig. 3C, upper panel). In marked contrast, western analysis of MV CD147 expression revealed (Fig. 3C, lower panel) only the high glycosylated form of the molecule, known to be essential for CD147 bioactivity $[35,36]$.

\section{Immunophenotypic characterization of MM- derived MVs}

We next studied if MM-derived MVs expressed additional biologically relevant receptors that are present on the surface of MM cells. Beginning with three representative HMCLs, Figure 4 again shows that MVs are enriched for CD147 expression as compared to the other molecules assayed. CD28 was present in 2 of the 3 HMCL-derived MVs as was CD45, albeit only slightly. We did not find evidence of the IGF-IR or CD138, a specific PC marker found on both normal and malignant cells, in the MVs. We next wished to characterize MVs isolated from patient plasma. In results not shown, we used Nanoparticle Tracking Analysis to determine the size and concentration of MVs found in normal and patient BM plasma. MVs harvested from normal BM and MGUS patient BM plasma contained an average of $1.1 \times 10^{9}$

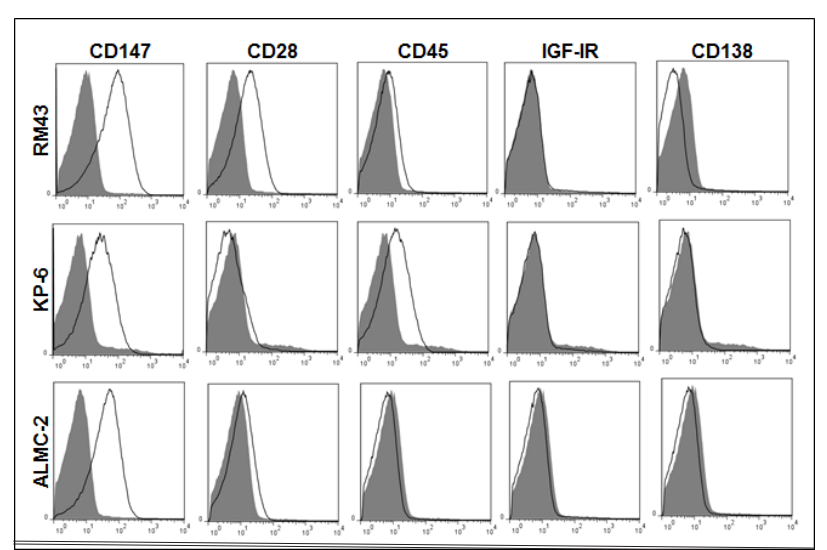

Figure 4: HMCL MV phenotypic analysis. Annexin- $\mathrm{V}+$ HMCL MVs were identified and assessed for CD28, CD147, CD138, CD45 and IGF-IR by flow cytometry (open histogram), revealing CD147 enrichment as compared to other markers in panel. Isotype (shaded histogram) used as a control. and $5.7 \times 10^{9}$ MVs per $\mathrm{mL}$, respectively. By contrast, the number of MVs isolated from MM BM plasma was approximately 4-fold higher $\left(2.6 \times 10^{10} \mathrm{MVs}\right.$ per $\left.\mathrm{mL}\right)$. Although a sizeable difference was noted in the MV concentration, there was no difference in average MV size across the three patient groups. Thus, MVs from normal BM plasma were on average $101 \mathrm{~nm}$, while MGUS MVs were measured at $102 \mathrm{~nm}$ followed by MM plasma MVs averaging $92 \mathrm{~nm}$ (data not shown). We next analyzed the immunophenotype of MVs from 6 representative MM patients and Figure 5A shows that they were all annexin- $\mathrm{V}+$ as well as $\mathrm{CD} 147+$. Because platelets and endothelial cells also express CD147, it was possible that the CD147+ MVs in BM plasma derived from either or both of these cell types. However, as shown in Figure 5A, the MVs largely lacked expression of CD31 and CD42,
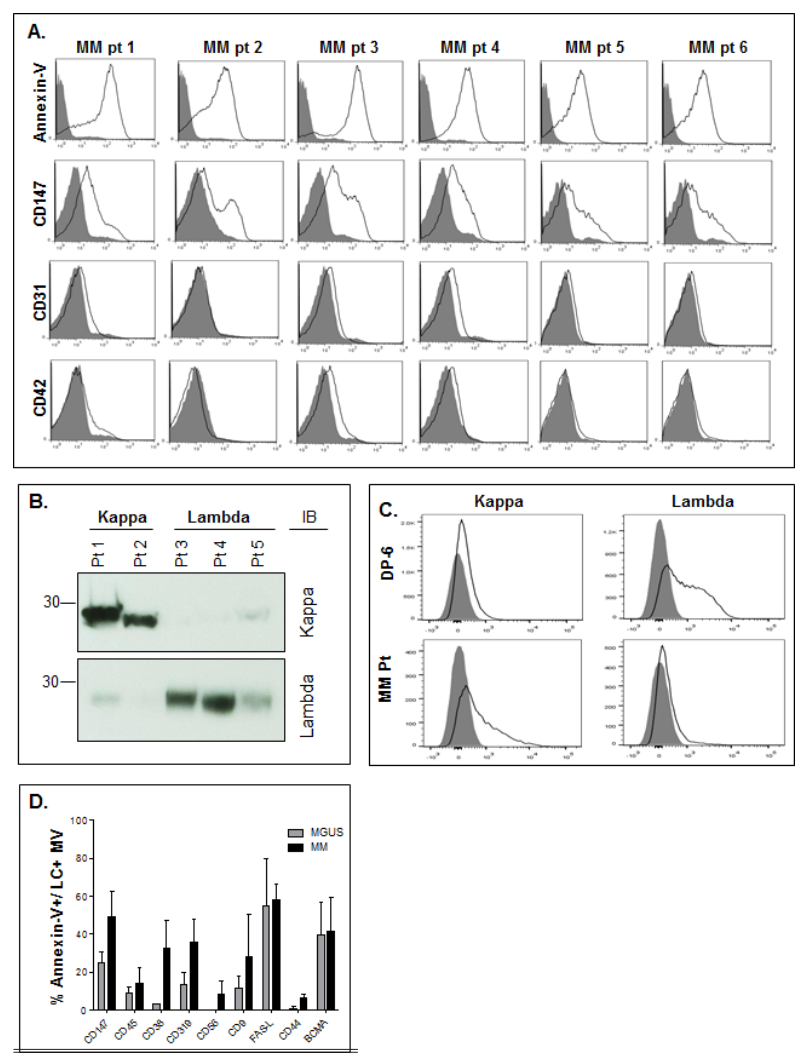

Figure 5: MM platelet free plasma MV characterization. A) Annexin-V+ MVs harvested from MM platelet free BM plasma were assessed for CD147 levels and CD31 and CD42 (open histograms) as compared to an isotype control (shaded histogram). B) Western blotting of platelet free BM plasma MVs harvested from 2 kappa ( $\kappa$ ) and 3 lambda $(\lambda)$ MM patients identifies LCs as a component of the MVs. C) Flow cytometry of DP- 6 cell line $(\lambda-$ restricted) and MM pt ( $\kappa-$ restricted) showing positivity of the appropriate LCs in MVs (open histogram) as compared to isotype control (shaded histogram). D) Platelet free BM plasma MV content of MM patients assessed for expression of a panel of markers. Data represent the percentage of dually stained annexin- $\mathrm{V}+$ and LC-restricted MVs from the patient samples which contain the indicated molecule. 
molecules known to be present on endothelial cell and platelet-derived MVs, respectively.

We next tested the ability of tumor cell Ig light chains (LC) to specifically mark MM cell-derived MVs. Precedence for this approach was provided by findings in the literature that urinary exosomes in amyloidosis patients contain LCs restricted to the LC displayed by the malignant PCs [37]. Thus, we isolated MVs from platelet free plasma from $5 \mathrm{MM}$ patients (2 patients with kappa LCs and 3 patients with lambda LCs) and performed western blot analysis. Figure 5B shows that all 5 samples had an expected $25 \mathrm{kDa}$ band corresponding to the LC

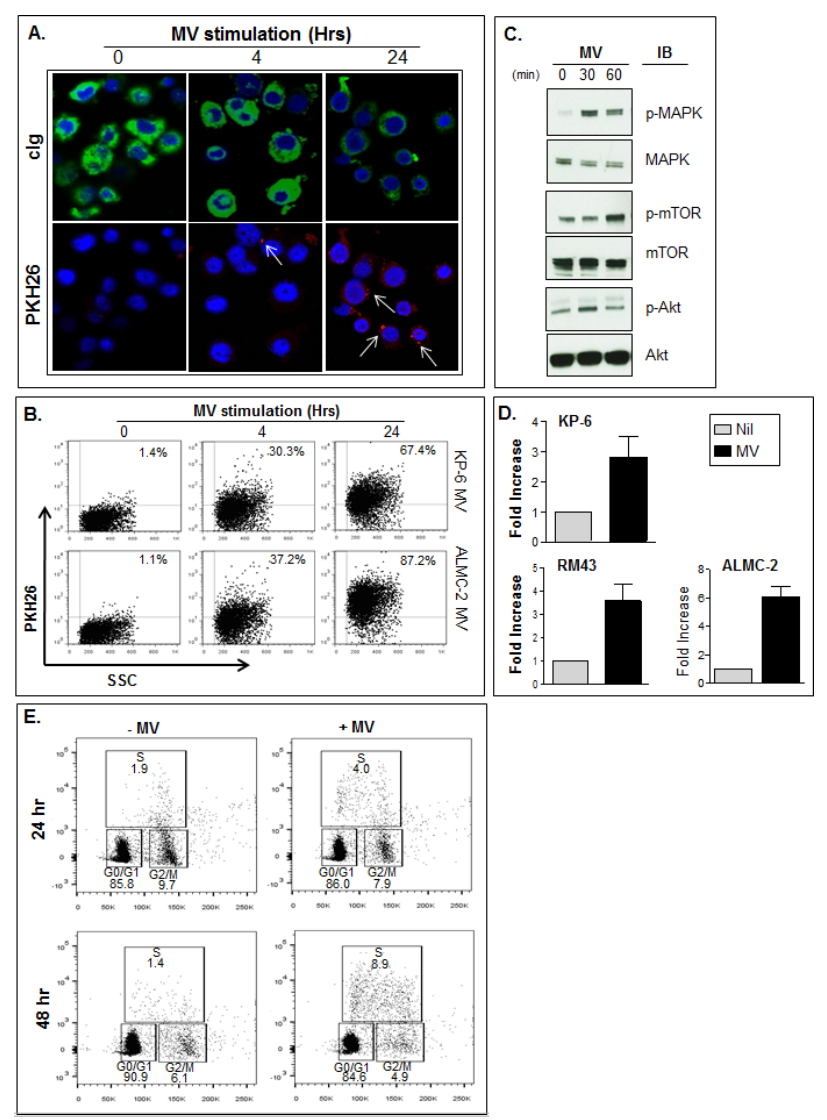

Figure 6: MVs promote HMCL proliferation. A) Confocal microscopy revealing internalization of PKH26 stained ALMC-2 MVs (red) indicated by white arrows in RM43 cells after 4 and $24 \mathrm{hr}(50 \mu \mathrm{g} / \mathrm{ml})$. RM43 cells visualized using cytoplasmic Ig (green) and DAPI (blue) counterstain. B) Flow cytometry revealing uptake of both PKH26 stained KP-6 and ALMC-2 MVs $(50 \mu \mathrm{g} / \mathrm{ml})$ in RM43 cells at 4 and $24 \mathrm{hr}$ time points. C) Western blot showing MV induced phosphorylation of MAPK, mTOR and Akt pathways at 30 and 60 minutes following $50 \mu \mathrm{g}$ MV stimulation. D) HMCLs KP-6, RM43 and ALMC-2 were used to assess the activity of ALMC-2 MVs. Proliferation was assessed by ${ }^{3} \mathrm{H}-\mathrm{TdR}$ incorporation after cells were cultured for $72 \mathrm{hrs}$ under unstimulated (Nil) conditions or with $50 \mu \mathrm{g} / \mathrm{mL}$ MVs. Results are displayed as fold increase over background. E) BrdU incorporation as shown by flow cytometry reveals HMCL LYMM transiting the cell cycle after stimulation of $50 \mu \mathrm{g} / \mathrm{ml} \mathrm{ALMC}-2 \mathrm{MVs}$ at both 24 and $48 \mathrm{hrs}$. monomer and that the MV LC signal precisely matched the patient tumor cell LC isotype. We also assessed MM patient-derived MVs by flow cytometry and Figure 5C reveals that MVs from the lambda LC-expressing DP-6 HMCL only react with anti- $\lambda$ and not anti- $\kappa$ antibodies whereas MVs from the representative $\kappa$-expressing MM patient sample were $\kappa$-positive and $\lambda$-negative. These results demonstrated the utility of this tool in specifically identifying patient plasma MVs that are highly likely to be released by the clonal tumor cells, thereby permitting specific phenotypic analysis of the MM-derived MVs. We next analyzed BM plasma MVs from 2 MGUS and $3 \mathrm{MM}$ patients and first identified annexin-V+ MVs that also expressed the patient specific LC isotype, thus allowing us to gate on MVs exclusively derived from the PC clone. Using this approach, we were able to demonstrate that CD147 was present on average in $\sim 50 \%$ of MM PCderived MVs, while only $25 \%$ of MGUS LC-positive MVs contained CD147 (Figure 5D). Higher percentages of LC-restricted MVs from MM cells expressed CD38, CD319 (FAS), CD44, and CD9 as compared with MVs from MGUS patients.

\section{HMCL MVs induce proliferation}

We next examined the biological activity of MM cell derived MVs and began using the HMCLs because of the lack of potentially confounding non-MM cells. As a first step, we determined if MVs could be internalized by the HMCLs. HMCL-derived MVs were stained with the membrane dye PKH26 prior to culturing with ALMC2 cells for 4 and 24 hrs. Confocal microscopy (Fig. 6A) demonstrates the accumulation and uptake of MVs by cells stained for cytoplasmic immunoglobulin. Likewise, flow cytometry shows RM43 cells possess the ability to incorporate fluorescently labeled MVs derived from both KP-6 and ALMC-2 cells (Fig. 6B), with levels of uptake increasing over time. Figure $6 \mathrm{C}$ shows by western blot that incubation of HMCL MVs with RM43 cells at 30 minutes induced both MAPK and Akt activation while mTOR phosphorylation was observed at 60 minutes. On the basis of our findings, we next examined whether MVinduced activation of these pathways resulted in HMCL proliferation. As expected, all HMCLs tested were growth responsive to IL-6, albeit to differing degrees (data not shown). Indeed, addition of HMCL-derived MVs from common collections reproducibly increased DNA synthesis in all HMCLs. MV stimulation revealed a 2.8, 3.8 , and 6-fold increase in proliferation over background of KP-6, RM43 and ALMC-2 cells respectively (Fig. 6D). Moreover, cell cycle analysis added further support that MVs enhance HMCL progression through the cell cycle (Fig. 6E). 


\section{CD147 enriched patient plasma MVs enhance HMCL proliferation}

Given that we had determined by flow cytometry that MVs obtained from MM platelet free plasma are enriched for CD147 we next sought to further confirm this finding by quantifying MV CD147 levels in a separate and distinct panel of MVs isolated from 8 MGUS and 14 MM platelet free BM plasma by ELISA. Figure 7A clearly shows that MM patients have MVs with significantly higher levels of CD147 than those obtained from MGUS samples. Although this assay does not allow us to
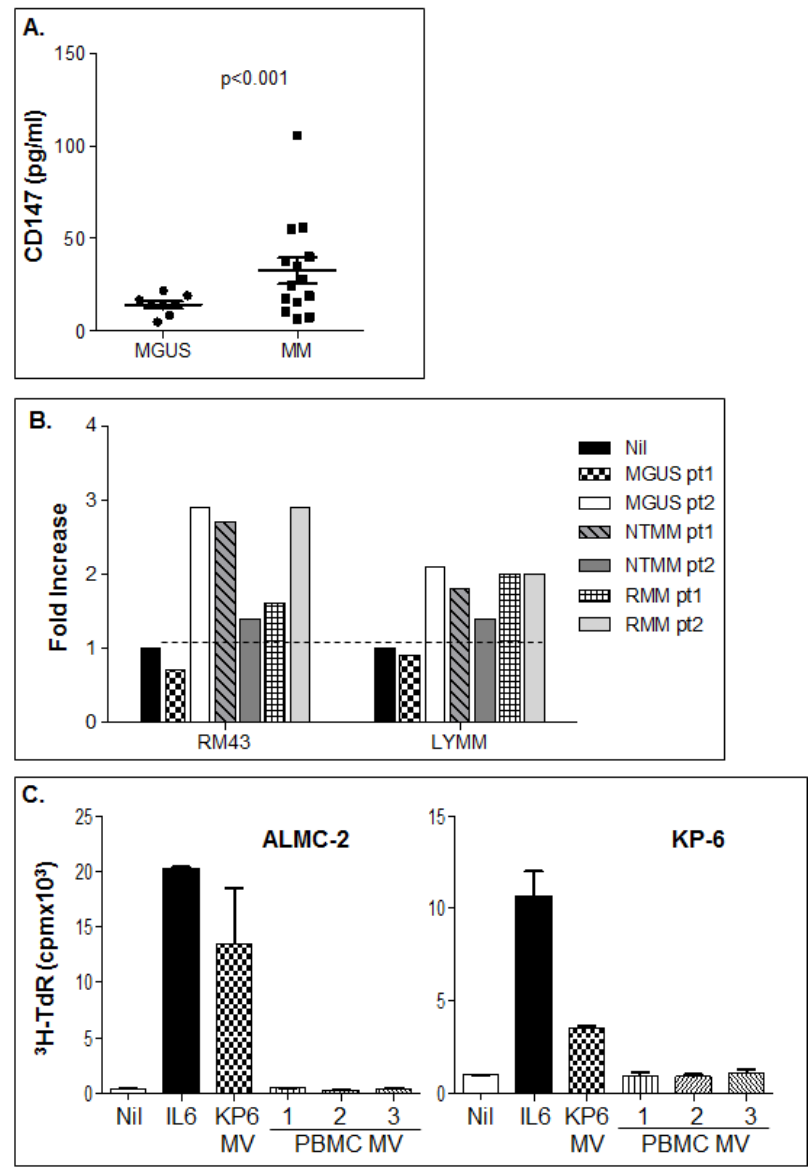

Figure 7: Bioactivity of MVs prepared from plateletfree bone marrow patient plasma. A) BM patient platelet free plasma derived MVs from MGUS (left panel) and MM pt samples (right panel) were assessed for CD147 protein levels by ELISA. B) HMCLs RM43 and LYMM were used to assess the activity of various MV samples $(50 \mu \mathrm{g} / \mathrm{ml})$ from MGUS, NTMM and RMM patients. Proliferation was assessed on day 3 by ${ }^{3} \mathrm{H}-\mathrm{TdR}$ incorporation and results displayed as fold increase over background. C) HMCL MVs but not normal PBMCderived MVs induce HMCL proliferation. ALMC-2 (left panel) and KP-6 (right panel) cells were incubated for $72 \mathrm{hrs}$ in the presence of MVs $(50 \mu \mathrm{g} / \mathrm{ml})$ obtained from either HMCL KP-6 cells or normal donor peripheral blood mononuclear cells. IL-6 $(1 \mathrm{ng} / \mathrm{ml})$ was used as a positive control and proliferation was assessed by ${ }^{3} \mathrm{H}-\mathrm{TdR}$ incorporation. definitively conclude that all of the detected CD147 was MM cell-derived, it does confirm our previous findings that $\mathrm{CD} 147$ is upregulated during disease progression and furthermore, allows us to postulate that CD147 may be a component of BM MVs in MM patients. Because of these results, we next determined whether MM patient plasma MVs also have the ability to enhance MM cell proliferation. MVs were harvested from the platelet free BM plasma from 2 MGUS, 2 never treated MM (NTMM), and 2 RMM patients. The bioactivity of these MV samples was assessed using the RM43 and LYMM HMCLs (Fig. 7B). Indeed, while one MGUS MV sample lacked activity, the second MGUS sample stimulated both HMCLs. MVs from the NTMM and RMM patients displayed variable levels of activity when assessed on the RM43 and LYMM HMCLs. Finally, to address the possibility that non-MM cell-derived MVs were stimulatory in this assay, we isolated MVs from normal peripheral blood mononuclear cells (PBMC) and compared their activity with HMCLderived MVs. Of note, PBMCs produced on average onetenth of the amount of MVs released by MM cells. Figure $7 \mathrm{C}$ clearly shows the lack of activity of PBMC MVs. Overall, these data suggest that tumor-derived MVs may

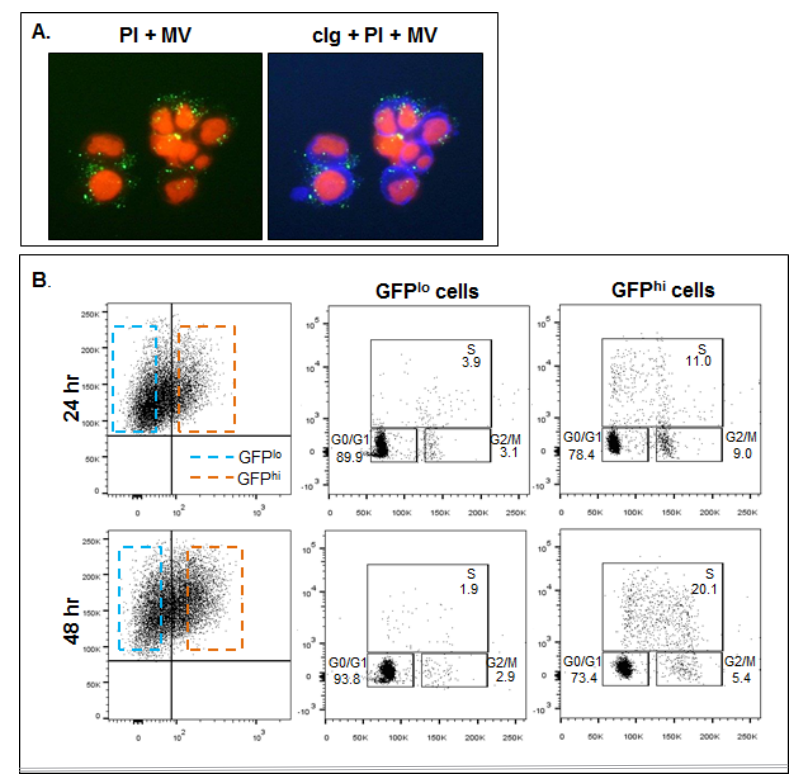

Figure 8: HMCL uptake of CD147 enriched MVs. A) Incubation of ALMC-2 cells (blue, cIg stain; right panel) at a concentration of $2 \times 10^{6} / \mathrm{ml}$ in IMDM+ $0.5 \%$ BSA with $50 \mu \mathrm{g} /$ $\mathrm{ml}$ ALMC-2 derived CD147 ${ }^{\mathrm{GFP}}$ MVs (green) for $24 \mathrm{hrs}$ revealed CD147 cellular uptake as assessed by immunofluorescence. PI was used as a counterstain. B) ALMC-2 cells were cultured at a concentration of $2 \times 10^{6} / \mathrm{ml}$ in IMDM $+0.5 \%$ BSA $+/-50 \mu \mathrm{g} /$ $\mathrm{ml}$ ALMC-2 derived CD147 ${ }^{\mathrm{GFP}}$ MVs for 24 and $48 \mathrm{hrs}$. After incubating for $4 \mathrm{hrs}$ at $37^{\circ} \mathrm{C}$ in the presence of BrdU, cell cycle analysis was performed. Cells incorporating higher levels of CD147 (GFPhi; orange dashed box) were shown to be enriched in both $\mathrm{S}$ and $\mathrm{G} 2 / \mathrm{M}$ phases at both time points (right panels) as compared to cells which incorporated less CD147 (GFPlo; blue dashed box ). 
be a novel mechanism by which various members of the MM clone are stimulated to proliferate in vivo.

\section{MVs promote proliferation of HMCLs through a CD147-mediated mechanism}

The last series of experiments were performed to determine if there is an association between MV-induced proliferation and CD147. We first transfected the ALMC2, RM43, and KP-6 HMCLs with a CD147 $7^{\text {GFP }}$ construct and MVs were collected from stably transfected cells. Figure 8A demonstrates by immunofluorescence that culturing MVs containing CD147 ${ }^{\text {GFP }}$ with ALMC-2 cells for 48 hours resulted in cellular uptake of CD147 ${ }^{\mathrm{GFP}}$ MVs. Because MVs drive HMCLs through the cell cycle (Fig. $6 \mathrm{E}$ ), to assess whether CD147 specifically contributed to this result, we incubated ALMC-2 cells with CD147 $7^{\text {GFP }}$ containing MVs for 24 and $48 \mathrm{hrs}$. FACS analysis revealed CD147 incorporation by GFP fluorescence. GFPhi and $\mathrm{GFP}^{\mathrm{lo}}$ populations were gated and subjected to cell cycle analysis. Figure $8 \mathrm{~B}$ shows there were significantly more GFP $^{\text {hi }}$ cells in $\mathrm{S}$ phase at both time points than $\mathrm{GFP}^{\mathrm{lo}}$ cells.

To further complement these studies and to
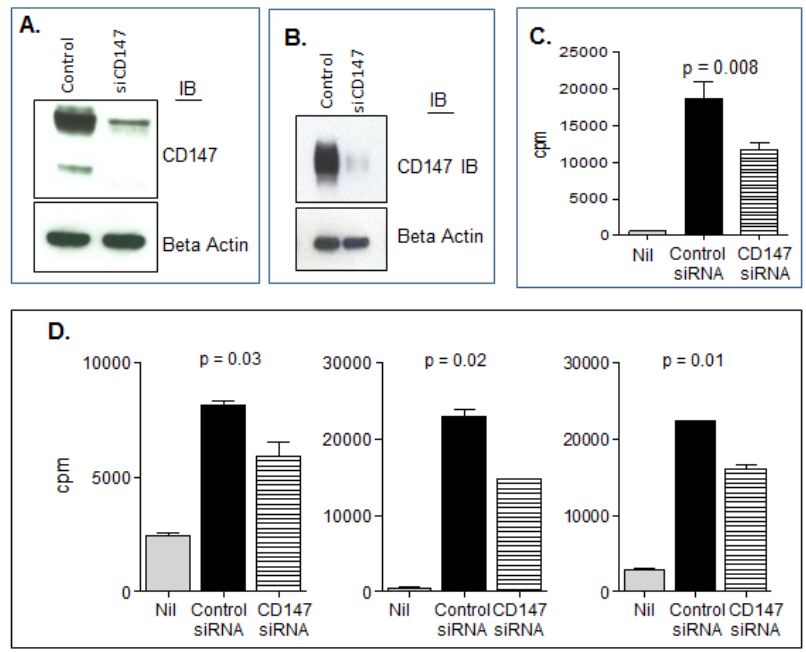

Figure 9: CD147 plays a role in MV bioactivity. A) Western blot verifying CD147 knockdown in ALMC-2 whole cell lysates. Beta actin used as a loading control. B) Western blot confirming downregulation of CD147 in ALMC-2 derived MVs. $72 \mathrm{hrs}$ after transfection of control siRNA or CD147 specific siRNA, MVs were collected. C) Downregulation of CD147 in HMCL-derived MVs as compared to mock transfected MVs decreased the proliferative response observed in ALMC-2 cells measured after 3 days of culture in IMDM $+0.5 \mathrm{BSA}+/-50 \mu \mathrm{g} /$ mL MVs. D) MVs were obtained from the KP-6 and ALMC-2 HMCLs 48-72 hours after transfection of either control or CD147 specific siRNA. Untreated KP-6 and ALMC-2 cells were then cultured in the presence of $10 \mu \mathrm{g}$ of control or CD147 siRNA MVs. Proliferation was assessed by $\left[{ }^{3} \mathrm{H}\right]$ thymidine incorporation after 72 hours. Proliferation of KP-6 cells with KP-6 MVs (left panel); ALMC-2 wth ALMC-2 MVs (middle panel), and KP-6 cells with ALMC-2 MVs (right panel). strengthen our conclusion of the relationship of CD147associated MVs and MM proliferation, we assessed the activity of MVs obtained from the HMCL KP-6 that had undergone siRNA-mediated downregulation of CD147. Figure 9A demonstrates by western blot that CD147 protein levels were successfully decreased in cell lysates as well as in MVs isolated from CD147 silenced KP-6 cells (Figure 9B). Multiple HMCLs were then cultured with MVs obtained from either control siRNA transfected cells or CD147 siRNA transfected cells and proliferation was assessed. Consistent with previous proliferation results, MVs from control siRNA transfected cells were able to enhance HMCL DNA synthesis, however, MVs collected from CD147 downregulated cells were significantly less effective (Figure 9C). Similar results were observed when MVs obtained from CD147 siRNA treated KP-6 and ALMC-2 cells were incubated with either the HMCL from which the MVs were isolated or mixed in a heterologous manner (Figure 9D). When incubating HMCLs with MVs harvested from CD147 siRNA transfected cells, western blotting revealed a decrease in MAPK and mTOR phosphorylation at 30 minutes as compared to those harvested from control siRNA transfected cells (Figure 10). AKT phosphorylation appeared unchanged. These

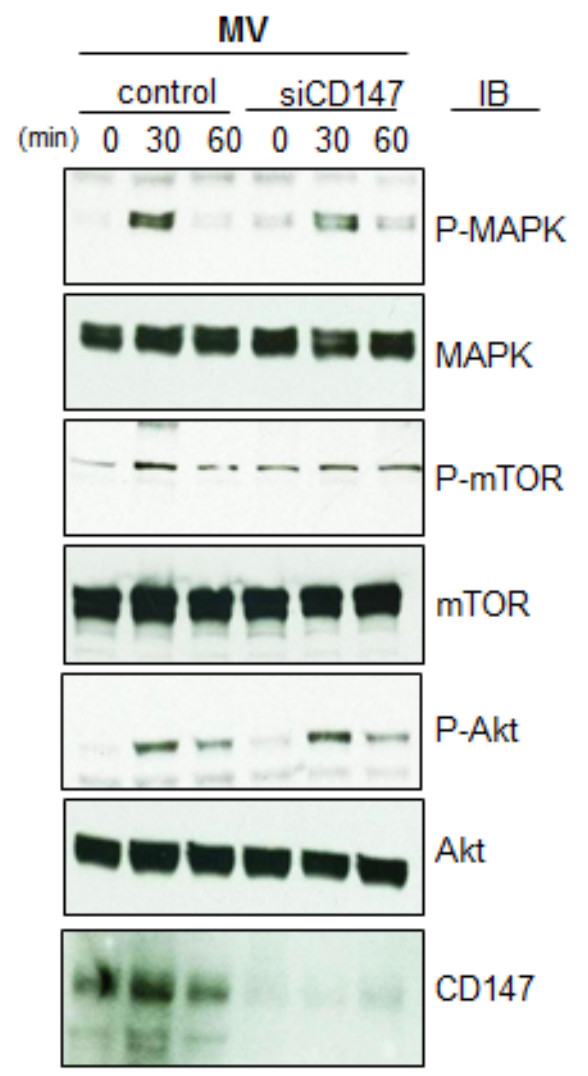

Figure 10: Downregulation of MV CD147 effect on signaling pathways. Western blot assessing the ability of MVs isolated from cells transfected with control siRNA or CD147 siRNA to activate MAPK, mTOR and Akt pathways in ALMC-2 cells. Cells were stimulated for 30 min with $50 \mu \mathrm{g}$ of MVs. 
results add support to the conclusion that the stimulatory activity of HMCL MVs can be attributed at least in part to CD147.

\section{DISCUSSION}

Previous work in the MM field has demonstrated that the interaction of malignant PCs with BM stromal cells is essential for survival of the abnormal PC clone [5, 6]. Specifically, MM genetic alterations have been shown to modify the biology of BM stromal cells (i.e., altered adhesion molecule expression and cytokine production), which effectively conditions the microenvironment to provide optimal support of malignant PC survival and proliferation. Thus, MM is an ideal model to study the effects of the tumor microenvironment on disease progression. Notably, there is currently a growing body of literature demonstrating that important aspects of tumor microenvironment interactions stem from the dysregulated release of bioactive MVs by tumor cells. The interaction of MVs and the transfer of their bioactive content to cells in the tumor microenvironment have been shown to have profound consequences on tumor growth and metastasis. Despite the fact that numerous publications in recent years have demonstrated MV shedding from tumor cells, this area of investigation is still considered relatively novel, especially in the field of MM [11]. In the current study, we sought to determine whether primary MM patient samples and HMCLs shed bioactive MVs. Indeed, all MM patient samples and HMCLs tested were found to shed MVs. Given that shedding of MVs generally increases following activation of pathways involved in cell stimulation, stress, or oncogenesis [38], it could be speculated that the level of MV release may increase across the disease spectrum from MGUS to SMM to MM. Further studies examining whether the level of MV shedding correlates with the disease status and aggressiveness are currently underway.

In addition to our demonstration that MM cells shed MVs, we also provide provocative evidence that MM-derived MVs are internalized by MM cells and that they have a feedback effect on the MM tumor cells themselves as revealed by their ability to stimulate signaling pathways associated with cell growth and to enhance MM cell proliferation. Our observations are consistent with prior publications showing that MVs have the ability to stimulate tumor cell growth through the transfer of bioactive molecules [39-41]. In this regard, all HMCL-derived MVs were found to induce proliferation in all HMCLs tested. Both a homotypic and heterotypic approach was utilized to ensure that the proliferative potential was not limited to one cell line or from a particular harvest of MVs. While MVs obtained from platelet free BM plasma from MGUS, NTMM and RMM patients varied in their ability to induce HMCL proliferation, strong conclusions regarding these findings were not able to be drawn due to the small number of patient samples tested. However, one explanation for the variable responsiveness observed in the MGUS samples is that the MGUS platelet free BM plasma derived MVs that did not elicit a response were from a patient with stable disease status whereas the MVs from the MGUS sample that induced a proliferation response is from a patient who is beginning to progress to either SMM or MM. Serial samples are necessary to formally test this speculation and beyond the scope of the current work.

Immunoglobulin rearrangement results in PCs becoming either kappa or lambda LC restricted and malignant PCs generally produce an overabundance of free LC. Notably, we determined that MM-derived MVs contain the LC expressed by the malignant PC clone. Detection of MV-associated LC by flow cytometry suggests that the LC is potentially present on the MV surface. Although this observation seems counterintuitive given the secreted nature of free LCs, there is precedence in the literature for free LC assemblies to form on the surface of MM cells [42]. Regarding MM, the presence of LCs in MM-derived MVs is a novel finding. Additionally, this observation establishes a potentially innovative tool for identifying and monitoring malignant PC-derived MVs in patient plasma over the disease spectrum from MGUS to SMM to MM.

In prior studies [25], we demonstrated that MM cells overexpress CD147 and is involved in MM cell proliferation. In this study we extend our prior work by showing that this molecule is present in MVs released by MM cells, and that it plays a role in MV-mediated stimulation of MM cell proliferation. Thus, MVs from all MM patient samples and HMCLs were found to be enriched for CD147. Although CD147 is known to be heterogeneous in terms of its N-glycosylation status, MMderived MVs only contained the more highly glycosylated version of CD147. This form of CD147 is the more bioactive molecule with a greater capacity to induce various biological responses such as induction of MMP production $[28,35,43]$. Using HMCLs transfected with a CD147-GFP construct, we generated HMCL MVs which contained fluorescently labeled CD147. We first showed that $\mathrm{CD} 147^{\mathrm{GFP}} \mathrm{MVs}$ associated with the malignant PCs. This result was consistent with what we observed using dye-labeled MVs; however, use of the CD $147^{\text {GFP }} \mathrm{MVs}$ is more rigorous in testing association and internalization due to the known pitfalls using dye-labeled MVs [41]. Because of CD147 homophilic interactions [28, 44], the observed association between CD147 ${ }^{\text {GFP }}$ MVs and the ALMC-2 cells suggested that CD147 may serve as one possible route of intercellular communication. Indeed, MM cells that internalized the greatest amount of CD147 ${ }^{\mathrm{GFP}} \mathrm{MVs}$ were greatly stimulated to enter the $\mathrm{S}$ phase of the cell cycle, whereas MM cells that only displayed low uptake of CD147 $7^{\mathrm{GFP}} \mathrm{MVs}$ were largely restricted within G0/G1. Moreover, when we downregulated CD147 in the HMCLs, not only was surface CD147 diminished, but CD147 
contained within MVs was also greatly reduced. This reduction of CD147 within the vesicles adversely affected their ability to stimulate proliferation of the HMCLs. Taken together these results support a critical role for CD147-enriched MVs in MM cell proliferation.

Our observations that MM-derived MVs have apparent auto-stimulatory activity on MM cells are very intriguing. Although MM is a clonal PC disorder, there is well-known heterogeneity within the clonal PCs. Therefore, we speculate that MM-derived MVs may provide a means by which more proliferative members within the clone can augment the proliferation and/or survival of less proliferative tumor cells. In support of this concept, in unpublished studies (Arendt and Jelinek), we have shown that single cell cloning of MM cells gives rise to populations of cells with differing growth rates and cytokine responsiveness. Finally, it is important to acknowledge that MM-derived MVs are highly likely to have biological effects on other cell types in the microenvironment. It is also probable that the CD147 molecule in MVs contributes to this activity as well given the known ability of CD147 to enhance angiogenesis and stimulate extracellular matrix degradation [28, 29]. Indeed, studies of this nature are currently underway.

An important question that remains to be answered is whether MVs play a role in metastasis in MM patients. In patients diagnosed with the disease, it is not uncommon to observe early focal tumor involvement in MM. However, with disease progression tumor cells somehow gain the ability to thrive in distant locations either within the bone marrow or in extramedullary sites resulting in the appearance of systemic intramedullary and extramedullary lesions. It is interesting to consider the hypothesis that bone marrow-resident MM cells release MVs into the circulation thereby creating pre-metastatic niches in other anatomic locations that attract and/or nurture the expansion of metastatic PCs. Consistent with this model, circulating PCs have been described in MM patients [45] and such levels correlate with both SMM and MM disease outcome. This hypothesis is consistent with studies in other malignancies including lung, breast, melanoma, and colon cancer, where it has been shown that tumorderived MVs have the ability to modify the local tumor microenvironment and contribute to metastatic spread of disease [46-49].

The findings described in this study significantly enhance our understanding of intercellular communication in $\mathrm{MM}$ by demonstrating that MM cells release biologically active MVs. Our findings that these MVs are enriched in CD147 expression and stimulate MM cell proliferation support the hypothesis that MM-derived MVs play a significant role in disease progression in the monoclonal gammopathies. Towards this end, we have begun to study MV levels and their correlation with MM disease progression as well as their ability to promote multiple biological activities that directly affect tumor cells and the tumor microenvironment including induction of angiogenesis and MMP production, and immunosuppression.

\section{MATERIAL AND METHODS}

\section{Patient material}

MGUS and MM patient BM aspirates were collected as part of the routine clinical examination. BM aspirates obtained from patients undergoing spine surgeries without coincident B lineage malignancies served as a source of normal BM PCs. The Mayo Clinic Institutional Review Board approved the protocol to obtain samples from healthy donors as well as from individuals with monoclonal gammopathies. Written informed consent to participate in this research study was provided by all subjects from whom BM aspirates were drawn in accordance with the Declaration of Helsinki. Normal donor peripheral blood (PB) served as the normal B cell source. BM aspirate mononuclear cells (MNCs) as well as PBMCs were isolated by Ficoll-Paque density gradient centrifugation. PCs were isolated from patient BM aspirates by magnetic bead separation using the human CD138 positive selection kit and a Robosep Cell Separator (StemCell Technologies, Vancouver, Canada).

\section{Cell lines and culture medium}

The HMCLs ANBL-6 [50], ALMC-2 [51], KP-6 [52], DT6I, LYMM, MCG, DP-6 [52],and RM43 were all derived in our laboratory while OCI-MY5 was obtained from ATCC (Manassas, VA, USA). HMCLs were maintained in IMDM supplemented with 5\% heatinactivated FBS (PAA Laboratories, Etobiocoke, Ontario, Canada) and $1 \mathrm{ng} / \mathrm{ml}$ recombinant IL-6, generously provided by Novartis (Basal, Switzerland).

\section{MV culture/isolation}

HMCLs and patient cells were washed in sterile saline, resuspended in IMDM + exo-free FBS (SBI, Mountain View, CA, USA) at $2 \times 10^{6} \mathrm{cells} / \mathrm{ml}$, and cultured for the indicated times at $37^{\circ} \mathrm{C}$. After incubation, the cell/media suspension was centrifuged at $1300 \mathrm{rpm}$ for 10 minutes at $4^{\circ} \mathrm{C}$ to remove cells. The supernatant was then centrifuged at $2500 \mathrm{xg}$ for 10 minutes at $4^{\circ} \mathrm{C}$, and again at $17,000 \mathrm{xg}$ for 45 minutes at $4^{\circ} \mathrm{C}$. Collected MVs were resuspended in DPBS, centrifuged at $17,000 \times \mathrm{xg}$ 2 more times and resuspended in sterile filtered PBS before quantifying using a BCA protein assay (Pierce, Rockford, IL, USA). MM patient platelet free plasma was obtained from BM aspirates by centrifuging at $2500 \mathrm{xg}$ for 
20 minutes at room temperature (RT) two times prior to isolating MVs.

\section{Immunophenotypic analysis}

Cells were stained using previously described flow cytometry methods [51]. MVs were resuspended in Hanks/ Hepes buffer and stained for 30 minutes at RT using various antibodies. To immunophenotype the MVs, the following antibodies were used: PE-conjugated CD147 (Abcam, Cambridge, MA, USA), PE-conjugated mouse IgG1 isotype control, VEGF R2 and IGF-IR (R\&D, Minneapolis, MN, USA), FITC-conjugated kappa and lambda (BD Biosciences, San Diego, CA, USA), PEconjugated CD138, CD45, CD38, CD28, CD44, CD9, CD56 (BD Biosciences), PE-conjugated FASL (Molecular Probes, Grand Island, NY, USA), PE-conjugated BCMA (Biolegend, San Diego, CA, USA), PE-conjugated CD319 (E-Bioscience, San Diego, CA, USA), APC- conjugatedAnnexin-V (BD Biosciences), and FITC-conjugated and PE-conjugated Annexin-V (Invitrogen, Grand Island, NY, USA). After incubation, 1\% paraformaldehyde was added to each sample along with the addition of $1 \mu \mathrm{M}$ TRUCount beads (BD Biosciences, San Diego, CA, USA) (diluted in $1 \% \mathrm{PF}$ ) per manufacturer's instructions prior to analysis on BD FACSCanto flow cytometer (BD Biosciences). Sample collection was completed after acquiring 1250 TRUCount bead events. MV size was verified using a submicron bead calibration kit (Bang Laboratories, Fishers, IN, USA). Data were analyzed with FlowJo software (Tree Star, Ashland, OR, USA). MVs are defined as events $\leq 1 \mu \mathrm{M}$ in diameter and positive for annexin-V.

\section{Nanoparticle tracking analysis}

The presence, size distribution, and concentration of vesicles was assessed by nanoparticle tracking analysis using a NanoSight NS300 instrument (NanoSight Ltd., Amesbury, UK). MV samples were diluted with PBS at a range of concentrations between $4 \times 10^{8}$ and $8 \times 10^{8}$ particles per milliliter. Each sample was loaded into a flow-cell top-plate using a syringe pump and three videos of 30 seconds were recorded and analyzed by NanoSight software (NTA 2.3.5 B16).

\section{Electron microscopy (EM)}

For scanning EM (SEM), samples were suspended in a fixative comprised of $4 \%$ paraformaldehyde, $1 \%$ glutaraldehyde in phosphate buffered saline, $\mathrm{pH} 7.2$ (Trumps), dried through a graded ethanol series, criticalpoint dried, mounted and sputter-coated for 90 seconds with gold palladium. The samples were then analyzed using a Hitachi S-4700 Field Emission Scanning Electron microscope; 0-30kV (Hitachi High Technologies America, Inc., Pleasanton, CA, USA). For transmission EM (TEM), samples were fixed in Trumps fixative, scraped from ACLAR ${ }^{\circledR}$ film and placed into $2 \%$ low melting agar. Samples were stained with $1 \%$ osmium tetroxide and $2 \%$ uranyl acetate, dehydrated through an ethanol series and embedded into Embed 812 resin. Following a $24 \mathrm{hr}$ polymerization at $60^{\circ} \mathrm{C}, 0.1 \mu \mathrm{M}$ ultrathin sections were post-stained with lead citrate. Micrographs were acquired using a JEOL 1400 TEM (JEOL, Tokyo, Japan). For immunolabeling EM (IEM), samples were collected and stained with anti-CD147 antibody (Genetex, Irvine, $\mathrm{CA}, \mathrm{USA}$ ) for $30 \mathrm{~min}$ at $4^{\circ} \mathrm{C}$ in $\mathrm{PBS}+2 \% \mathrm{MV}$-depleted FCS. Cells were then washed in PBS $3 \mathrm{X}$ and stained with colloidal gold conjugated anti-rabbit Ig (Amersham, Piscataway, NJ, USA) for $30 \mathrm{~min}$ at $4^{\circ} \mathrm{C}$ followed by 3 washes using PBS. After the last wash, samples were silver enhanced and fixed in Trumps fixative. Samples were then processed following the TEM protocol.

\section{DNA synthesis assays}

DNA synthesis assays measuring $\left[{ }^{3} \mathrm{H}\right]$-thymidine (TdR) incorporation (Perkin Elmer, Waltham, MA) were performed as previously described [52].

\section{Cell cycle and DNA content analysis}

HMCLs were incubated in complete IMDM media containing $10 \mu \mathrm{M}$ bromodeoxyuridine (BrdU) overnight prior to staining the next day using a BrdU-APC flow kit (BD Biosciences) as previously described [53]. MV incorporation was analyzed and cell cycle analysis was determined on $\mathrm{GFP}^{\text {hi }}$ and $\mathrm{GFP}^{\mathrm{lo}}$ cell populations using the FACStar (BD Biosciences). Data were analyzed using FlowJo software (Tree Star, Ashland, OR, USA).

\section{CD147 ELISA}

CD147 was assayed by a conventional sandwich based ELISA following the manufacturer's protocol (Sinobiologicals, Bejing, China). Captured CD147 was visualized and absorbance readings were made at $450 \mathrm{~nm}$, using a 96-well EPOCH microplate spectrophotometer (Biotek, Winooski, VT, USA). CD147 levels in samples were determined by interpolation from a standard curve.

\section{Western blot analysis}

Cells lysates were subjected to $10 \%$ SDS-PAGE and CD147 probing was performed as previously described [25]. Blots were probed with one or more of the following antibodies: CD147 (GeneTex) at a 1:2500 dilution, phospho MAPK or total MAPK at a 1:1000 dilution (NEB, 
Ipswich, MA, USA), phospho mTOR or total mTOR at a 1:1000 dilution (Millipore), kappa or lambda (Southern Biotech, Birmingham, Alabama, USA), or $\beta$-actin (Novus, Littleton, CO, USA) at a 1:5000 dilution. HRP-conjugated secondary antibodies (GE Healthcare, Fairfield, CT, USA) were used at a 1:2000 dilution.

\section{Labeling of MVs and ALMC-2 with PKH fluorescence}

MVs were labeled with a PKH26 red fluorescent labeling kit (Sigma-Aldrich, St. Louis, MO, USA) per manufacturer's instructions. ALMC-2 cells were stained for cytoplasmic Ig using FITC-conjugated polyclonal antihuman $\operatorname{Ig}(\mathrm{H}+\mathrm{L})$ antibody (BD Biosciences) after fixing with $95 \%$ ethanol for 5 minutes. Slides were washed in PBS for 5 minutes prior to mounting with Vectashield containing DAPI (Vector Laboratories, Burlingame, CA, USA) and viewed by confocal microscopy (Zeiss LSM 510 confocal laser-scanning microscope, Zeiss, Thornwood, NY, USA). Images were acquired using an Olympus DP71 microscope digital camera equipped with Olympus DP manager software (Olympus Imaging America, Center Valley, PA, USA).

\section{CD147-GFP fusion construct}

To generate the CD147-GFP fusion construct, the full-length CD147 isoform 1 (also known as Basigin-2) cDNA [54], including the 5' Kozak sequence and the ATG initiation codon but not the 3' stop codon, was amplified from cDNA synthesized from RNA isolated from the ALMC-2 cells. The forward PCR primer (5'-GGCCGCGAATTCATGGCGGCTGCGCTGTTC-3') was designed to include an XhoI site while the reverse primer (5'-GTCCGCCAGAGGAACTCTT CCCGGGATCCATCA-3') was engineered to include a BamHI site to facilitate in-frame cloning into the pEGFP-N1 vector (Clontech, Mountain View, CA, USA). The construct was validated by Sanger sequencing. To establish stable cell lines that permanently express the CD147-GFP fusion protein, the construct was linearized using the ApaLI restriction enzyme and then transfected into ALMC-2, RM43, and KP-6 cells by electroporation. Five days after transfection, GFP-expressing cells were sorted on an ARIA II FACS sorter (BD Biosciences). The expression of the fusion protein was validated by FACS analysis and Western blotting (data not shown).

\section{siRNA transfection parameters}

CD147/BSG siGenome siRNA duplexes were purchased from Dharmacon Research, Inc. (Lafayette, CO, USA). Transfection of the siRNA duplexes was achieved via electroporation as previously described [25, 55]. Following the transfection period, MVs were harvested from cells as described above.

\section{Statistical analysis}

Statistical analysis was performed using a Wilcoxon rank test. Values of $p$ less than 0.05 were considered significant.

\section{ACKNOWLEDGEMENTS}

The authors wish to thank Dr. Gregory Gores for allowing us access to his NanoSight NS300 instrument and to Dr. Petra Hirsova and Mr. Nathan Werneburg for their assistance in using this instrument to size and quantitate microvesicles. This work was supported by the National Institutes of Health (CA164204 and CA164232; awarded to DFJ).

\section{Author contributions}

BKA and DKW designed and performed research, analyzed data, and wrote the manuscript. XW and RCT performed research and analyzed data. DFJ designed research and wrote and approved the manuscript. All the authors reviewed and gave final approval of the manuscript.

\section{Editorial note}

This paper has been accepted based in part on peerreview conducted by another journal and the authors' response and revisions as well as expedited peer-review in Oncotarget

\section{Conflict of interest}

The authors declare no conflict of interest related to this work.

\section{REFERENCES}

1. Kyle RA, Rajkumar SV. Multiple myeloma. N Engl J Med. 2004; 351: 1860-1873.

2. Criteria for the classification of monoclonal gammopathies, multiple myeloma and related disorders: a report of the International Myeloma Working Group. Br J Haematol. 2003; 121: 749-757.

3. Kyle RA, Rajkumar SV. Monoclonal gammopathy of undetermined significance. Br J Haematol. 2006; 134: 573589.

4. Kumar SK, Rajkumar SV, Dispenzieri A, Lacy MQ, 
Hayman SR, Buadi FK, Zeldenrust SR, Dingli D, Russell SJ, Lust JA, Greipp PR, Kyle RA, Gertz MA. Improved survival in multiple myeloma and the impact of novel therapies. Blood. 2008; 111: 2516-2520.

5. Ghobrial IM. Myeloma as a model for the process of metastasis: implications for therapy. Blood. 2012; 120: 2030 .

6. Morgan GJ, Walker BA, Davies FE. The genetic architecture of multiple myeloma. Nat Rev Cancer. 2012; 12: 335-348.

7. Ratajczak J, Wysoczynski M, Hayek F, JanowskaWieczorek A, Ratajczak MZ. Membrane-derived microvesicles: important and underappreciated mediators of cell-to-cell communication. Leukemia. 2006; 20: 1487 1495.

8. van Doormaal FF, Kleinjan A, Di Nisio M, Buller HR, Nieuwland R. Cell-derived microvesicles and cancer. Neth J Med. 2009; 67: 266-273.

9. Al-Nedawi K, Meehan B, Kerbel RS, Allison AC, Rak J. Endothelial expression of autocrine VEGF upon the uptake of tumor-derived microvesicles containing oncogenic EGFR. Proc Natl Acad Sci U S A. 2009; 106: 3794-3799.

10. Baran J, Baj-Krzyworzeka M, Weglarczyk K, Szatanek R, Zembala M, Barbasz J, Czupryna A, Szczepanik A. Circulating tumour-derived microvesicles in plasma of gastric cancer patients. Cancer Immunol Immunother. 2010; 59: 841-850.

11. Skog J, Wurdinger T, van Rijn S, Meijer DH, Gainche L, Sena-Esteves M, Curry WT, Jr., Carter BS, Krichevsky AM, Breakefield XO. Glioblastoma microvesicles transport RNA and proteins that promote tumour growth and provide diagnostic biomarkers. Nat Cell Biol. 2008; 10: 1470-1476.

12. Piccin A, Murphy WG, Smith OP. Circulating microparticles: pathophysiology and clinical implications. Blood Rev. 2007; 21: 157-171.

13. Valenti R, Huber V, Filipazzi P, Pilla L, Sovena G, Villa A, Corbelli A, Fais S, Parmiani G, Rivoltini L. Human tumor-released microvesicles promote the differentiation of myeloid cells with transforming growth factor-betamediated suppressive activity on $\mathrm{T}$ lymphocytes. Cancer Res. 2006; 66: 9290-9298.

14. Deregibus MC, Cantaluppi V, Calogero R, Lo Iacono M, Tetta C, Biancone L, Bruno S, Bussolati B, Camussi G. Endothelial progenitor cell derived microvesicles activate an angiogenic program in endothelial cells by a horizontal transfer of mRNA. Blood. 2007; 110: 2440-2448.

15. Cocucci E, Racchetti G, Meldolesi J. Shedding microvesicles: artefacts no more. Trends Cell Biol. 2009; 19: 43-51.

16. Al-Nedawi K, Meehan B, Rak J. Microvesicles: messengers and mediators of tumor progression. Cell Cycle. 2009; 8: 2014-2018.

17. D'Souza-Schorey C, Clancy JW. Tumor-derived microvesicles: shedding light on novel microenvironment modulators and prospective cancer biomarkers. Genes Dev. 2012; 26: 1287-1299.

18. Ghosh AK, Secreto CR, Knox TR, Ding W, Mukhopadhyay D, Kay NE. Circulating microvesicles in B-cell chronic lymphocytic leukemia can stimulate marrow stromal cells: implications for disease progression. Blood. 2010; 115: 1755-1764.

19. Pap E, Pallinger E, Falus A. The role of membrane vesicles in tumorigenesis. Crit Rev Oncol Hematol. 2011; 79: 213223.

20. Sun L, Wang HX, Zhu XJ, Wu PH, Chen WQ, Zou P, Chen C. Serum deprivation elevates the levels of microvesicles with different size distributions and selectively enriched proteins in human myeloma cells in vitro. Acta Pharmacologica Sinica. 2014;35:381-193.

21. Liu Y, Zhu XJ, Zeng C, Wu PH, Wang HX, Chen ZC, Li QB. Microvesicles secreted from human multiple myeloma cells promote angiogenesis. Acta Pharmacologica Sinica. 2014;35:230-238.

22. Harshman SW, Canella A, Ciarlariello PD, Rocci A, Agarwal K, Smith EM, Talabere T, Efebera YA, Hofmeister CC, Benson DM, Jr., Paulaitis ME, Freitas MA, Pichiorri F. Characterization of multiple myeloma vesicles by label-free relative quantitation. Proteomics. 2013; 13: 3013-3029.

23. Millimaggi D, Mari M, D’Ascenzo S, Carosa E, Jannini EA, Zucker S, Carta G, Pavan A, Dolo V. Tumor vesicleassociated CD147 modulates the angiogenic capability of endothelial cells. Neoplasia. 2007; 9: 349-357.

24. Sidhu SS, Mengistab AT, Tauscher AN, LaVail J, Basbaum C. The microvesicle as a vehicle for EMMPRIN in tumorstromal interactions. Oncogene. 2004; 23: 956-963.

25. Arendt BK, Walters DK, Wu X, Tschumper RC, Huddleston PM, Henderson KJ, Dispenzieri A, Jelinek DF. Increased expression of extracellular matrix metalloproteinase inducer (CD147) in multiple myeloma: role in regulation of myeloma cell proliferation. Leukemia. 2012; 26: 22862296.

26. Iacono KT, Brown AL, Greene MI, Saouaf SJ. CD147 immunoglobulin superfamily receptor function and role in pathology. Exp Mol Pathol. 2007; 83: 283-295.

27. Tang Y, Nakada MT, Kesavan P, McCabe F, Millar H, Rafferty P, Bugelski P, Yan L. Extracellular matrix metalloproteinase inducer stimulates tumor angiogenesis by elevating vascular endothelial cell growth factor and matrix metalloproteinases. Cancer Res. 2005; 65: 3193-3199.

28. Sun J, Hemler ME. Regulation of MMP-1 and MMP2 production through CD147/extracellular matrix metalloproteinase inducer interactions. Cancer Res. 2001; 61: 2276-2281.

29. Zhu P, Lu N, Shi ZG, Zhou J, Wu ZB, Yang Y, Ding J, Chen ZN. CD147 overexpression on synoviocytes in rheumatoid arthritis enhances matrix metalloproteinase production and invasiveness of synoviocytes. Arthritis Res Ther. 2006; 8: R44. 
30. Jayachandran M, Miller VM, Heit JA, Owen WG. Methodology for isolation, identification and characterization of microvesicles in peripheral blood. $\mathrm{J}$ Immunol Methods. 2012; 375: 207-214.

31. Dey-Hazra E, Hertel B, Kirsch T, Woywodt A, Lovric S, Haller H, Haubitz M, Erdbruegger U. Detection of circulating microparticles by flow cytometry: influence of centrifugation, filtration of buffer, and freezing. Vasc Health Risk Manag. 2010; 6: 1125-1133.

32. Shah MD, Bergeron AL, Dong JF, Lopez JA. Flow cytometric measurement of microparticles: pitfalls and protocol modifications. Platelets. 2008; 19: 365-372.

33. van der Pol E, Coumans F, Varga Z, Krumrey M, Nieuwland R. Innovation in detection of microparticles and exosomes. J Thromb Haemost. 2013; 11 Suppl 1: 36-45.

34. Egawa N, Koshikawa N, Tomari T, Nabeshima K, Isobe T, Seiki M. Membrane type 1 matrix metalloproteinase (MT1MMP/MMP-14) cleaves and releases a 22-kDa extracellular matrix metalloproteinase inducer (EMMPRIN) fragment from tumor cells. J Biol Chem. 2006; 281: 37576-37585.

35. Tang W, Chang SB, Hemler ME. Links between CD147 function, glycosylation, and caveolin-1. Mol Biol Cell. 2004; 15: 4043-4050.

36. Jia L, Zhou H, Wang S, Cao J, Wei W, Zhang J. Deglycosylation of CD147 down-regulates Matrix Metalloproteinase-11 expression and the adhesive capability of murine hepatocarcinoma cell HcaF in vitro. IUBMB Life. 2006; 58: 209-216.

37. Ramirez-Alvarado $\mathrm{M}$, Ward CJ, Huang $\mathrm{BQ}$, Gong $\mathrm{X}$, Hogan MC, Madden BJ, Charlesworth MC, Leung N. Differences in immunoglobulin light chain species found in urinary exosomes in light chain amyloidosis (Al). PLoS One. 2012; 7: e38061.

38. Rak J. Microparticles in cancer. Semin Thromb Hemost. 2010; 36: 888-906.

39. MacKenzie A, Wilson HL, Kiss-Toth E, Dower SK, North RA, Surprenant A. Rapid secretion of interleukin-1beta by microvesicle shedding. Immunity. 2001; 15: 825-835.

40. Baj-Krzyworzeka M, Szatanek R, Weglarczyk K, Baran J, Urbanowicz B, Branski P, Ratajczak MZ, Zembala M. Tumour-derived microvesicles carry several surface determinants and mRNA of tumour cells and transfer some of these determinants to monocytes. Cancer Immunol Immunother. 2006; 55: 808-818.

41. Pucci F, Pittet MJ. Molecular pathways: tumor-derived microvesicles and their interactions with immune cells in vivo. Clin Cancer Res. 2013; 19: 2598-2604.

42. Hutchinson AT, Malik A, Berkahn MB, Agostino M, To J, Tacchi JL, Djordjevic SP, Turnbull L, Whitchurch CB, Edmundson AB, Jones DR, Raison RL, Ramsland PA. Formation of assemblies on cell membranes by secreted proteins: molecular studies of free lambda light chain aggregates found on the surface of myeloma cells. Biochem J. 2013; 454: 479-489.
43. Tang W, Hemler ME. Caveolin-1 regulates matrix metalloproteinases-1 induction and CD147/EMMPRIN cell surface clustering. J Biol Chem. 2004; 279: 11112-11118.

44. Yu XL, Hu T, Du JM, Ding JP, Yang XM, Zhang J, Yang B, Shen X, Zhang Z, Zhong WD, Wen N, Jiang H, Zhu P, et al. Crystal structure of HAb18G/CD147: implications for immunoglobulin superfamily homophilic adhesion. J Biol Chem. 2008; 283: 18056-18065.

45. Billadeau D, Van Ness B, Kimlinger $T$, Kyle RA, Therneau TM, Greipp PR, Witzig TE. Clonal circulating cells are common in plasma cell proliferative disorders: a comparison of monoclonal gammopathy of undetermined significance, smoldering multiple myeloma, and active myeloma. Blood. 1996; 88: 289-296.

46. Ghasemi R, Grassadonia A, Tinari N, Piccolo E, Natoli C, Tomao F, Iacobelli S. Tumor-derived microvesicles: the metastasomes. Med Hypotheses. 2013; 80: 75-82.

47. Martins VR, Dias MS, Hainaut P. Tumor-cell-derived microvesicles as carriers of molecular information in cancer. Curr Opin Oncol. 2013; 25: 66-75.

48. Muralidharan-Chari V, Clancy JW, Sedgwick A, D'SouzaSchorey C. Microvesicles: mediators of extracellular communication during cancer progression. J Cell Sci. 2010; 123: 1603-1611.

49. Wysoczynski M, Ratajczak MZ. Lung cancer secreted microvesicles: underappreciated modulators of microenvironment in expanding tumors. Int J Cancer. 2009; 125: 1595-1603.

50. Jelinek DF, Ahmann GJ, Greipp PR, Jalal SM, Westendorf JJ, Katzmann JA, Kyle RA, Lust JA. Coexistence of aneuploid subclones within a myeloma cell line that exhibits clonal immunoglobulin gene rearrangement: clinical implications. Cancer Res. 1993; 53: 5320-5327.

51. Arendt BK, Ramirez-Alvarado M, Sikkink LA, Keats JJ, Ahmann GJ, Dispenzieri A, Fonseca R, Ketterling RP, Knudson RA, Mulvihill EM, Tschumper RC, Wu X, Zeldenrust SR, et al. Biologic and genetic characterization of the novel amyloidogenic lambda light chain-secreting human cell lines, ALMC-1 and ALMC-2. Blood. 2008; 112: 1931-1941.

52. Westendorf JJ, Ahmann GJ, Greipp PR, Witzig TE, Lust JA, Jelinek DF. Establishment and characterization of three myeloma cell lines that demonstrate variable cytokine responses and abilities to produce autocrine interleukin-6. Leukemia. 1996; 10: 866-876.

53. Walters $\mathrm{DK}, \mathrm{Wu} \mathrm{X}$, Tschumper RC, Arendt BK, Huddleston PM, Henderson KJ, Dispenzieri A, Jelinek DF. Evidence for ongoing DNA damage in multiple myeloma cells as revealed by constitutive phosphorylation of H2AX. Leukemia. 2011; 25: 1344-1353.

54. Liao CG, Kong LM, Song F, Xing JL, Wang LX, Sun ZJ, Tang H, Yao H, Zhang Y, Wang L, Wang Y, Yang XM, $\mathrm{Li}$ Y, et al. Characterization of basigin isoforms and the inhibitory function of basigin-3 in human hepatocellular 
carcinoma proliferation and invasion. Mol Cell Biol. 2011; 31: 2591-2604.

55. Walters DK, Jelinek DF. The effectiveness of doublestranded short inhibitory RNAs (siRNAs) may depend on the method of transfection. Antisense Nucleic Acid Drug Dev. 2002; 12: 411-418. 